\title{
Magnetospectroscopy of epitaxial few-layer graphene
}

\author{
M.L. Sadowski ${ }^{\text {a }}$ G. Martinez ${ }^{\text {a }}$, M. Potemski ${ }^{a}$, C. Berger ${ }^{\text {b,c }}$, \\ W.A. de Heer ${ }^{b}$ \\ ${ }^{a}$ Grenoble High Magnetic Field Laboratory, Grenoble,France \\ ${ }^{\mathrm{b}}$ Georgia Institute of Technology, Atlanta, Georgia, USA \\ ${ }^{\mathrm{c}}$ Institut Néel, CNRS, Grenoble, France
}

\begin{abstract}
The inter-Landau level transitions observed in far-infrared transmission experiments on few-layer graphene samples show a behaviour characteristic of the linear dispersion expected in graphene. This behaviour persists in relatively thick samples, and is qualitatively different from that of thin samples of bulk graphite.
\end{abstract}

Key words: Graphene, Cyclotron resonance, PACS: 71.70.Di, 76.40.+b, 78.30.-j, 78.67.-n

The interest in two-dimensional graphite is fuelled by its particular band structure and ensuing dispersion relation for electrons, leading to numerous differences with respect to "conventional" two-dimensional electron gases (2DEG). Single graphite layers (graphene) have long been used as a starting point in band structure calculations of bulk graphite [1,2,3] and, more recently, carbon nanotubes [4. The band structure of a single graphene sheet is considered to be composed of cones located at two inequivalent Brillouin zone corners at which the conduction and valence bands merge. In the vicinity of these points the electron energy depends linearly on its momentum, which implies that free charge carriers in graphene are governed not by Schrödinger's equation, but rather by Dirac's relativistic equation for zero rest mass particles, with an effective velocity $\tilde{c}$, which replaces the speed of light [5,6].

The recent appearance of ultrathin graphite layers (few-layer graphene, FLG), obtained by epitaxial [7,8,9] and exfoliation techniques [10], followed by single graphene and its unusual sequence of quantum Hall states [11,12] has re-ignited this interest. The prospects of studying quantum electrodynamics 


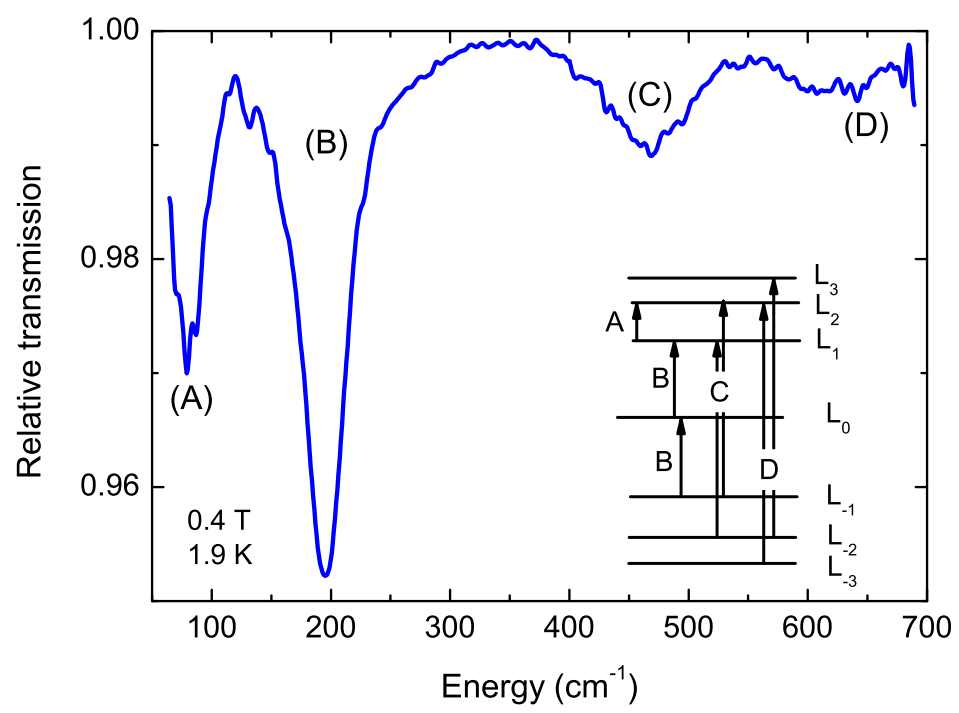

Fig. 1. Transmission spectrum of epitaxial graphene at $0.4 \mathrm{~T}$. The inset shows a schematic of the assignations of the observed transitions.

in solid state experiments on the one hand and the possibility of future applications in carbon-based electronics on the other are currently driving a considerable research effort. The majority of the published literature remains theoretical; the extremely small lateral dimensions $(\approx 10 \mu \mathrm{m})$ of the graphene flakes used in the above-mentioned transport experiments makes them difficult objects for experimental studies. Moreover, due to the somewhat hit-and-miss character of the exfoliation method, as well as the inherent difficulty of obtaining large numbers of samples, it appears to be an unlikely candidate for possible applications. Epitaxial methods on the other hand offer the opportunity of obtaining relatively large, high quality two-dimensional graphite [13]. In the following, we present optical measurements of the characteristic dispersion relation of FLG, confirming directly its linear ("relativistic") character.

A number of epitaxial graphene samples have been studied by means of farinfrared magnetotransmission measurements. The samples were about $4 \times 4$ $\mathrm{mm}^{2}$ in area, grown by sublimating $\mathrm{SiC}$ substrates at high temperatures 9,13]. The experimental details and part of the results have been described elsewhere [14.

A representative transmission spectrum of a three-graphene-layer sample is shown in Fig. 1 for a weak magnetic field of $0.4 \mathrm{~T}$. When the magnetic field is increased, all the features visible in this figure are displaced towards higher energies. Furthermore, their strength increases [14] and more features become visible at higher energies. The positions of the features observed for the sample containing 3 graphene layers are plotted versus the square root of the magnetic field in Fig. 2. It may be seen that the resonant energies observed evolve proportionally to the square root of the magnetic field. The oscillator strength 
Table 1

\begin{tabular}{lrr}
\hline Line & Slope in units of $\tilde{c} \sqrt{2 e \hbar}$ & Transition \\
\hline A & $\sqrt{2}-\sqrt{1}$ & $L_{1} \rightarrow L_{2}$ \\
$\mathrm{~B}$ & 1 & $L_{0} \rightarrow L_{1}\left(L_{-1} \rightarrow L_{0}\right)$ \\
$\mathrm{C}$ & $\sqrt{2}+\sqrt{1}$ & $L_{-1} \rightarrow L_{2}\left(L_{-2} \rightarrow L_{1}\right)$ \\
$\mathrm{D}$ & $\sqrt{3}+\sqrt{2}$ & $L_{-2} \rightarrow L_{3}\left(L_{-3} \rightarrow L_{2}\right)$ \\
$\mathrm{E}$ & $\sqrt{4}+\sqrt{3}$ & $L_{-3} \rightarrow L_{4}\left(L_{-4} \rightarrow L_{3}\right)$ \\
$\mathrm{F}$ & $\sqrt{5}+\sqrt{4}$ & $L_{-4} \rightarrow L_{5}\left(L_{-5} \rightarrow L_{4}\right)$ \\
$\mathrm{G}$ & $\sqrt{6}+\sqrt{5}$ & $L_{-5} \rightarrow L_{6}\left(L_{-6} \rightarrow L_{5}\right)$ \\
$\mathrm{H}$ & $\sqrt{7}+\sqrt{6}$ & $L_{-6} \rightarrow L_{7}\left(L_{-7} \rightarrow L_{6}\right)$ \\
$\mathrm{I}$ & $\sqrt{8}+\sqrt{7}$ & $L_{-7} \rightarrow L_{8}\left(L_{-8} \rightarrow L_{7}\right)$ \\
\hline
\end{tabular}

Observed lines and their assignments

of the transition labelled B in Fig 1 has also been shown [14] to increase linearly with the square root of the magnetic field.

These results are, in a first approximation, in excellent agreement with predictions arising from a simple single-particle model of non-interacting massless Dirac fermions.

Using appropriate graphene wavefunctions [4] and the Hamiltonian commonly used to describe electrons in a single graphene layer, it is fairly straightforward to work out the optical selection rules [15]. It may then be shown that the allowed transitions are $L_{n} \rightarrow L_{m}$ such that $|m|=|n|-1$ for the "+" circular polarisation and $|m|=|n|+1$ in the "-" circular polarisation. For unpolarised radiation, used in the current experiment, the allowed transitions are simply those between states $n, m$ such that $|m|=|n| \pm 1$. The Landau level energies are obtained as

$$
E_{n}=\tilde{c} \sqrt{2 \hbar e B|n|}
$$

where $\tilde{c}$ is the effective velocity of the Dirac fermions, B is the magnetic field and $n=0, \pm 1, \pm 2 \ldots$ is the Landau level index (the electron and hole levels being identical). The energies of the allowed optical transitions may then be concisely written as

$$
E_{n}^{t}=\tilde{c} \sqrt{2 \hbar e B}(\sqrt{|n+1|} \pm \sqrt{|n|})
$$

The positions of the transitions shown in Fig. 2 are summarised in Table 1. It should be stressed that all the positions of all the observed lines are described 


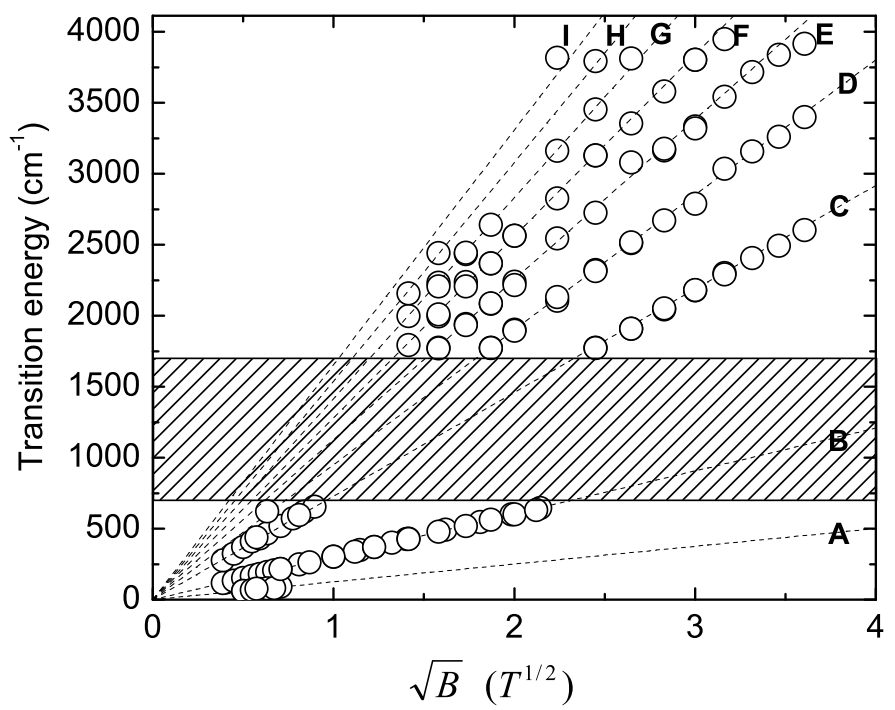

Fig. 2. Evolution with magnetic field of transitions observed in transmission. The letters correspond to those used in Fig. 1, the shaded region corresponds to the range where the substrate is opaque.

by a single fitting parameter - the effective light velocity $\tilde{c}$. We should add, for the sake of completeness, that the present experiment, using unpolarised light, does not distinguish between electrons and holes, which are expected to be identical in terms of the effective mass and dispersion relation. Thus, transition A, attributed to the $L_{1} \rightarrow L_{2}$ process, could also be due to the corresponding $L_{-2} \rightarrow L_{-1}$ one. While a p-type character appears to be unlikely, it cannot be ruled out on the basis of the experiment in question.

The striking agreement of the experimental data obtained using several layers of graphene with expectations for a single layer is surprising, given that calculations suggest a completely different behaviour already for a graphene bilayer [17. On the other hand, it has long been known that particles with a linear dispersion exist in bulk graphite as well - a minority pocket of carriers in the vicinity of the $\mathrm{H}$ point of the Brillouin zone were shown to give rise to electronic transitions following a square root dependence on the magnetic field [18. The question therefore is posed: at what point, if at all, does epitaxial FLG become bulk graphite?

Early work on epitaxial graphene [7] suggested that the process of baking $\mathrm{SiC}$ substrates led to a single graphene layer floating above a graphite layer. More recent calculations [22] suggest that the first carbon layer on top of an $\mathrm{SiC}$ substrate has an electronic structure different from that of graphene, and acts as a buffer, allowing subsequent layers to behave like graphene. A strong dependence of the electronic structure of FLG on the type of stacking has also been suggested [23]. The common Bernal, or AB, stacking found for example in HOPG graphite is usually assumed for all FLG structures as well; this is 


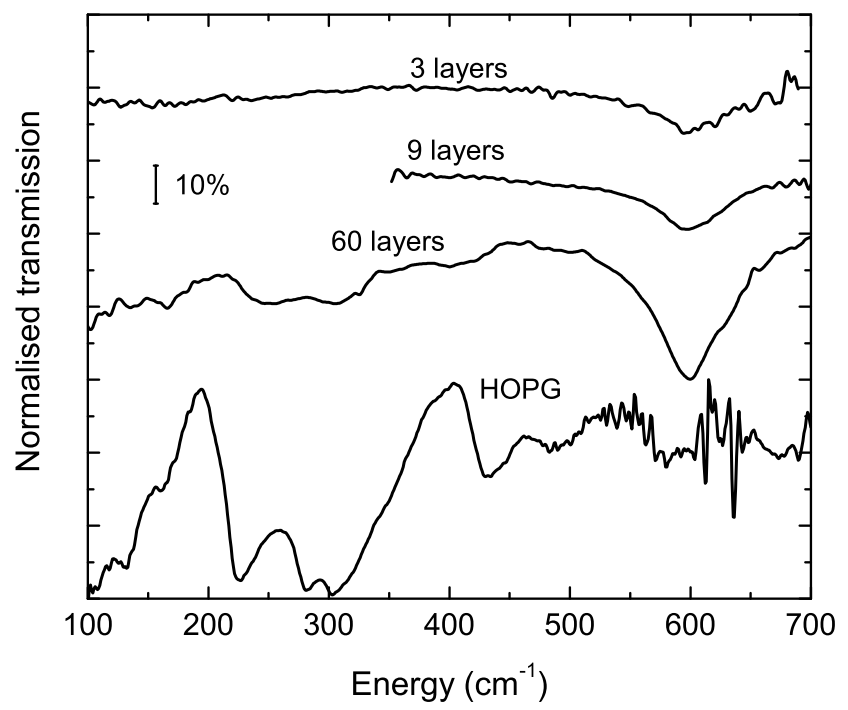

Fig. 3. Transmission spectra at $4 \mathrm{~T}$ for epitaxial FLG samples (top three) of varying thickness and, for comparison, of HOPG graphite at the same magnetic field.

not necessarily the case. Also, let us note that the HOPG interlayer distance of $3.354 \stackrel{\AA}{\mathrm{A}}$ may not be the correct value for epitaxial graphene.

In order to elucidate the effect of multiplying layers on the transmission spectrum, samples of varying thickness were studied and compared with a layer of HOPG obtained by exfoliation. The details of this study shall be presented elsewhere [19]; for the time being let us note the qualitative differences in the spectra, shown in Fig. 3. Four spectra are shown, at a magnetic field of 4T: for sample consisting of 3, 9 and 60 layers of graphene on $\mathrm{SiC}$, and for the HOPG sample. The dominant feature in the epitaxial samples is always the $L_{0} \rightarrow L_{1}$ $\left(L_{-1} \rightarrow L_{0}\right)$ transition; we can see that it grows stronger as the number of layers is increased, and is several times stronger for the sample containing 60 layers. In this sample one can also see the appearance of other features at lower energies, which were absent in the thinner samples, and which appear to correspond to bulk-like features visible in the lowest (HOPG) trace in the figure. On the other hand, the $L_{0} \rightarrow L_{1}\left(L_{-1} \rightarrow L_{0}\right)$ transition, which has a square root dispersion even in the 60 layer sample, is absent from the HOPG spectrum.

The observed persistence of the Dirac fermion-like behaviour of the carriers in epitaxial FLG up to relatively thick ( $19 \mathrm{~nm}$ ) structures appears to suggest that the structure of this material is in fact different from that of bulk HOPG. The simplest explanation would be a far weaker interaction between adjacent graphene layers, leading to a sequence of graphene layers instead of bulk, or even multilayer, graphene. More studies are necessary to elucidate this question. 
The GHMFL is a "Laboratoire conventionné avec l'UJF et l'INPG de Grenoble". The present work was supported in part by the European Commission through grant RITA-CT-2003-505474 and by grants from the Intel Research Corporation and the NSF: NIRT "Electronic Devices from Nano-Patterned Epitaxial Graphite".

\section{References}

[1] P.R. Wallace, Phys. Rev. 71, 622 (1947)

[2] J.W. McClure, Phys. Rev. 104, 666 (1956)

[3] J.C. Slonczewski and P.R. Weiss, Phys. Rev.109, 272 (1958)

[4] T. Ando, J. Phys. Soc. Jpn. 74, 777 (2005)

[5] F.D.M. Haldane, Phys. Rev. Lett. 61, 2015 (1988)

[6] Y. Zheng and T. Ando, Phys. Rev. B 65, 245420 (2002)

[7] I. Forbeaux, J.-M. Themlin, and J.-M. Debever, Phys. Rev. B 58, 16396 (1998)

[8] A.Charrier, A. Coati, T. Argunova, F. Thibaudau, Y. Garreau, R. Pinchaux, I. Forbeaux, J.-M. Debever, M. Sauvage-Simkin, J.-M. Themlin, J. Appl. Phys. 92, $2479(2002)$

[9] C. Berger, Z. Song, T. Li, X. Li, A.Y. Ogbazghi, R. Feng, Z. Dai, A.N. Marchenkov, E.H. Conrad, P.N. First, and W.A. de Heer, J. Phys. Chem. 108, 19912 (2004).

[10] K.S. Novoselov, A.K. Geim, S.V. Morozov, D. Jiang, Y. Zhang, S.V. Dubonos, I.V. Grigorieva, and A.A. Firsov, Science 306, 666 (2004)

[11] K.S. Novoselov, A.K. Geim, S.V. Morozov, D. Jiang, M.I. Katsnelson, I.V. Grigorieva, S.V. Dubonos, and A.A. Firsov, Nature 438, 197 (2005).

[12] Y. Zhang, Y.-W. Tan, H.L. Stormer and P. Kim, Nature 438, 201 (2005).

[13] C. Berger, Z. Song, T. Li, X. Li, X. Wu, N. Brown, C. Naud, D. Mayou, A.N. Marchenkov, E.H. Conrad, P.N. First, and W.A. de Heer, Science 312, 1191 (2006)

[14] M.L. Sadowski, G. Martinez, M. Potemski, C. Berger, and W.A. de Heer, Phys. Rev. Lett 97, 266405 (2006).

[15] M.L. Sadowski, G. Martinez, M. Potemski, C. Berger, and W.A. de Heer, Int. J. Mod. Phys. B, in press.

[16] V.P. Gusynin, S.G.Sharapov, and J.P. Carbotte, J. Phys.: Condens. Matter 19, 026222 (2007) 
[17] D.S.L. Abergel and V.I. Fal'ko, cond-mat/0610673

[18] W.W. Toy, M.S. Dresselhaus, and G. Dresselhaus, Phys. Rev. 15, 4077 (1977)

[19] M.L. Sadowski et al., to be published

[20] T. Ohta, A. Bostwick, T. Seyller, K. Horn, and E. Rotenberg, Science 313, 951 (2006)

[21] B. Partoens and F.M. Peeters, Phys. Rev. B 74,075404 (2006)

[22] F. Varchon, R. Feng, J. Hass, X. Li, B.N. Nguyen, C. Naud, P. Mallet, J.-Y. Veuillen, C. Berger, E.H. Conrad, and L. Magaud, cond-mat/0702311

[23] F. Guinea, A.H. Castro Neto, N.M.R. Peres, Phys. Rev. B 73, 245426 (2006) 